\title{
Risk of Adverse Cardiovascular Events Following a Myocardial Infarction in Patients Receiving Combined Clopidogrel and Proton Pump Inhibitor Treatment: A Nested Case-Control Study
}

\author{
Nawal Farhat ${ }^{1,2} \mathbb{D} \cdot$ Nicholas Birkett $^{1,2} \mathbb{( D} \cdot$ Nisrine Haddad $^{1,2}\left(\right.$ Yannick Fortin $^{2} \mathbb{D} \cdot$ Franco Momoli $^{1,3}$. \\ Shi Wu Wen ${ }^{1,4,5}$ - Andreas Wielgosz ${ }^{6}$ D $\cdot$ Doug S. McNair $^{7}$ (D) Donald R. Mattison ${ }^{1,2,3}$ (D) Daniel Krewski ${ }^{1,2,3}$ (D)
}

Published online: 2 July 2020

(c) The Author(s) 2020

\begin{abstract}
Background The clinical implications of potential interactions between proton pump inhibitors (PPIs) and clopidogrel have been debated for over a decade.

Objective We assessed the association between combined clopidogrel-PPI treatment and the risk of recurrent myocardial infarction (MI) and three secondary outcomes.

Patients and Methods A nested case-control study was conducted within Cerner Corporation's Health Facts ${ }^{\circledR}$ database. A retrospective cohort of patients who experienced a first MI and started clopidogrel treatment was created. Within this cohort, patients experiencing a second MI (cases) were matched with up to five controls. Logistic regression was used to estimate adjusted odds ratios (aORs). Findings were compared with those obtained from models with three negative control exposure drugs: $\mathrm{H}_{2}$ receptor antagonists, prasugrel, and ticagrelor.

Results In total, 2890 recurrent MI cases were identified within 12 months following entry into the cohort of clopidogrel users $(N=52,006)$. aOR for PPI use versus non-use among clopidogrel users was 1.08 [95\% confidence interval (CI) $0.95-1.23$ ]. Similar ORs were obtained for secondary endpoints. A positive association between combined use of clopidogrel/PPIs and increased risk of MI was seen in the group aged 80-89 years (aOR 1.26; 95\% CI 1.05-1.51). No associations with MI were observed for (1) H2 receptor antagonist use versus non-use among clopidogrel users or (2) PPI use versus non-use among prasugrel users or among ticagrelor users.

Conclusions Overall, our findings do not support a significant adverse clinical impact of concomitant clopidogrel/PPI use by patients with MI. Nonetheless, investigation of the possible association seen in those aged 80-89 years may be warranted.
\end{abstract}

Electronic supplementary material The online version of this article (https://doi.org/10.1007/s40801-020-00204-9) contains supplementary material, which is available to authorized users.

Nawal Farhat

nfarhat@uottawa.ca

1 School of Epidemiology and Public Health, University of Ottawa, Ottawa, ON, Canada

2 McLaughlin Centre for Population Health Risk Assessment, Ottawa, ON, Canada

3 Risk Sciences International, Ottawa, ON, Canada

4 Clinical Epidemiology Program, Ottawa Hospital Research Institute, Ottawa, ON, Canada

5 OMNI Research Group, Department of Obstetrics and Gynecology, University of Ottawa Faculty of Medicine, Ottawa, ON, Canada

6 The Ottawa Hospital, Ottawa, ON, Canada

7 Bill \& Melinda Gates Foundation, Seattle, WA, USA

\section{Key Points}

Clopidogrel and proton pump inhibitors are two medications that are commonly coprescribed to patients with acute coronary syndrome.

Findings do not support an increased risk of cardiovascular events among patients receiving both medications compared with patients only receiving clopidogrel.

Patients aged $>80$ years receiving both medications may be at a higher risk than those only receiving clopidogrel; this finding warrants further research. 


\section{Introduction}

Clopidogrel is a popular antiplatelet agent for reducing adverse cardiovascular events in patients with acute coronary syndrome (ACS) or those undergoing percutaneous coronary intervention (PCI) [1]. Since clopidogrel can increase the risk of gastrointestinal bleeding, treatment guidelines recommend the coprescription of a proton pump inhibitor (PPI) [2]. The potential for a drug-drug interaction between clopidogrel and PPIs has been investigated in numerous studies since 2009. Pharmacodynamic studies have noted that the antiplatelet activity of clopidogrel is diminished in the presence of PPIs because of competitive inhibition: PPIs are metabolized by hepatic cytochrome P450 (CYP) isoenzyme 2C19 (CYP2C19), which plays a major role in activating clopidogrel in the human body [3]. In 2009, the US FDA and other regulatory bodies issued warnings that concomitant treatment should be avoided. Since 2009, numerous epidemiological studies have arrived at inconclusive findings regarding the potential for adverse cardiovascular effects of combined treatment relative to treatment with clopidogrel only. We recently conducted a systematic review and meta-analysis [4] summarizing findings from 50 studies that examined the adverse cardiovascular events associated with clopidogrel and PPI use. Although the overall findings from our review did not provide strong support for a causal association, results from individual studies were inconsistent. The majority of the studies we reviewed analyzed administrative data, including insurance claims data and prescription databases. In the present study, we analyzed data from Cerner Health Facts ${ }^{\circledR}$, an electronic medical records (EMRs) database that has not been previously analyzed, to examine the potential clinical impact of the joint effects of clopidogrel and PPIs.

\section{Methods}

\subsection{Data Source}

Patients for this study were recruited from the Cerner Health Facts ${ }^{\circledR}$ database. Health Facts ${ }^{\circledR}$ consists of EMRs that include time-stamped information on admissions, discharges, diagnoses, hospital procedures, drug prescriptions, and laboratory tests. Over 500 US hospitals contributed data for over 69 million unique patients from 1 January 2000 to 31 December 2016. Healthcare facilities from all US census regions contribute to Health Facts ${ }^{\circledR}$. The healthcare facilities are classified by urban/rural setting, capacity (bed size), and teaching status. The majority of the data in Health Facts ${ }^{\circledR}(65 \%)$ are from academic medical centers. Only data on inpatient encounters were considered in this study since prescription data for outpatient encounters is incomplete. Health Facts ${ }^{\circledR}$ is compliant with the Insurance Portability and Accountability Act. The study protocol was approved by the Ottawa Health Science Network Research Ethics Board at The Ottawa Hospital, Canada.

\subsection{Cohort Selection}

We identified a retrospective cohort of patients who were hospitalized for a first MI (recorded in Health Facts ${ }^{\circledR}$ ) between 1 January 2001 and 31 December 2015 and received clopidogrel during the hospitalization. All inpatients and emergency room (ER) patients who were discharged alive were eligible for cohort entry $(n=95,562)$. Only the first qualifying encounter for each patient was considered. International Classification of Diseases, Ninth Revision (ICD-9) and Tenth Revision (ICD-10) codes (ICD9: 410.xx excluding 410. $\times 2$ and ICD-10 I21 $\mathrm{x}$ ) in the principal position (diagnosis chiefly responsible for the hospitalization) or secondary position (diagnosis priority $=2$ in the list of diagnoses for that encounter in the patients' records) [5] were used to identify those admitted with an MI. Patients aged 18-89 years at the time of hospitalization were eligible for inclusion if their age, sex, and ethnicity data were complete. Patients who received prasugrel or ticagrelor in addition to clopidogrel during the qualifying hospitalization were excluded to avoid including patients likely to switch antiplatelets $(n=79)$. Patients with a length of stay (LOS) $<3$ days during their first MI hospitalization were also excluded $(n=37,394)$ (Fig. 1$)$. We restricted cohort entry to patients with a minimum of 3-day hospitalization to ensure that cohort patients received chronic medications as pharmacy orders in the hospital; as such, we could use pharmacy orders data within Health Facts ${ }^{\circledR}$ to collect information on chronic medication use at baseline. The ICD codes used in addition to the minimum LOS of 3 days were previously reported to have a positive predictive value of $92.4 \%$ when used with the primary and secondary positions for MI diagnosis [6]. The date of hospital discharge of the qualifying encounter served as the date of cohort entry for each patient. Further, patients with adjacent encounters that occurred within $24 \mathrm{~h}$ were combined into one encounter; this could happen in two cases: (1) the patient was admitted to one hospital for MI and then transferred to another hospital or (2) the patient was admitted to the ER for an MI and was later admitted as an inpatient at the same hospital. 


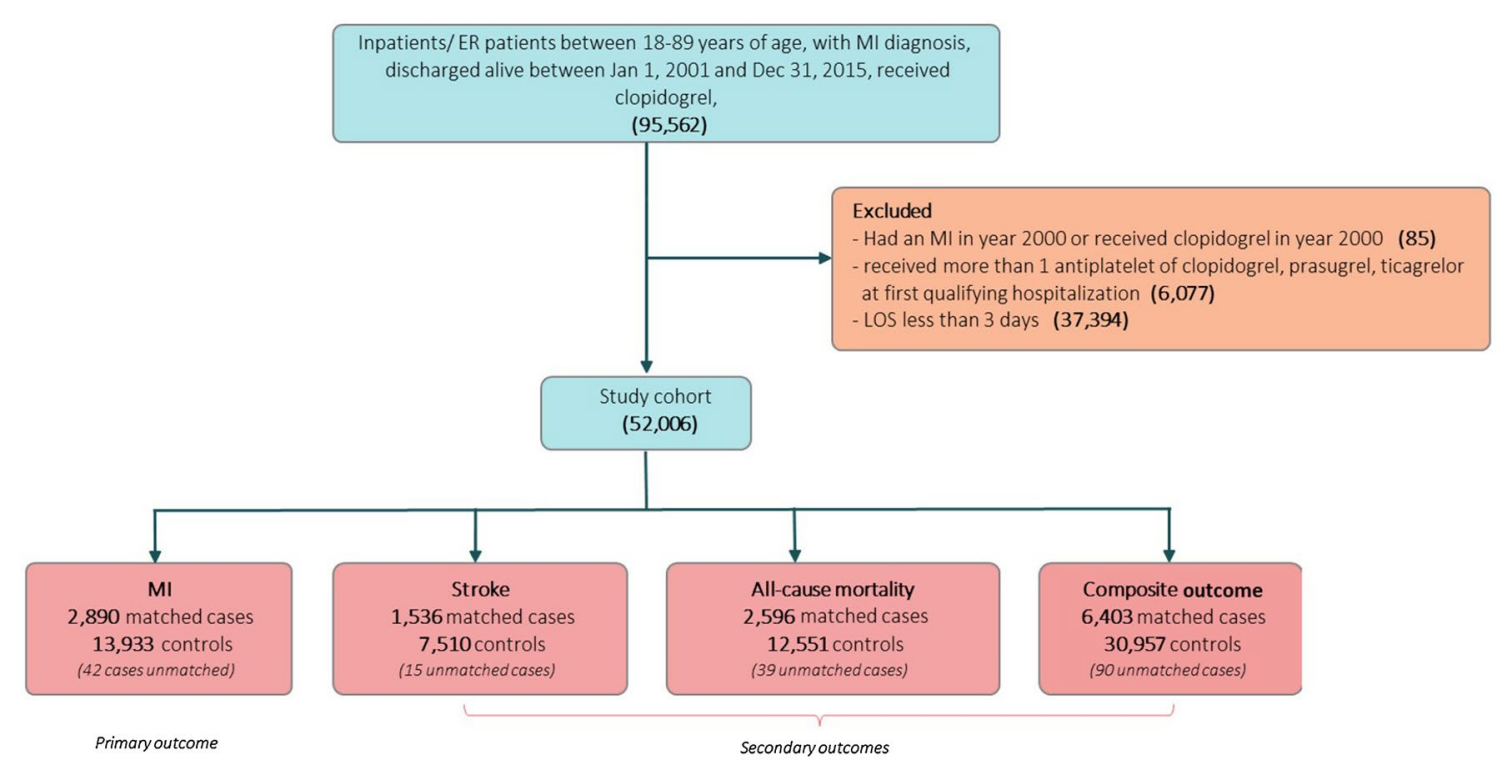

Fig. 1 Selection of study cohort and cases from Health Facts ${ }^{\circledR}$. ER emergency room, LOS length of stay, MI myocardial infarction

\subsection{Case Definition and Control Selection}

\subsubsection{Primary Outcome}

Cases were defined as members of the cohort who were hospitalized with an MI within 1 year of their cohort entry date. MI cases had to have an ICD code for MI (ICD9: 410.xx excluding 410. $\times 2$ and ICD-10 I21 $x$ ) in the principal or secondary position (diagnosis priority 1 or 2 in Health Facts ${ }^{\circledR}$ ) and hospitalization for at least 3 days (unless the patient died during the hospital encounter). Each case was assigned an index date corresponding to the date of hospitalization of the second MI.

Controls were selected using incidence density sampling. For each case, a risk set of potential controls was constructed. Candidate controls had the same sex (male/ female), same ethnicity (Caucasian, African American, other), and similar age as the corresponding case ( \pm 3 years). In addition, the controls had to have entered the cohort within 30 days of the matched case's cohort entry date. Matching on cohort entry was used to ensure cases and matched controls were treated in accordance with similar treatment guidelines, which changed over time because of FDA warnings regarding the potential interaction between PPIs and clopidogrel. Up to five controls were randomly selected for each case within each risk set. The risk set sampling methods employed allowed for a patient to serve as a control for multiple cases. Cases that could not be matched to at least one control were excluded from the analysis. Risk set sampling provides consistent risk estimates in nested case-control analyses $[7,8]$.

\subsubsection{Secondary Outcomes}

The secondary outcomes assessed were stroke, all-cause mortality, and the composite outcome (stroke, MI, and allcause mortality). Cases for the secondary outcomes were selected using a similar approach as for MI case selection. Patients who were admitted to the hospital within 1 year of cohort entry and had an ICD code for stroke as the primary diagnosis were identified as stroke cases. Patients who were readmitted during the 1-year post cohort entry for any reason and had a discharge disposition of death were identified as cases for the all-cause mortality endpoint. (As cause of death is not captured in HealthFacts, we were unable to examine cause-specific mortality.) For the composite outcome, patients who experienced any one of MI, stroke, or death from any cause were considered cases. For the composite outcome, only the earliest event after cohort entry was considered among patients who experienced more than one event. The date of admission for a case event was designated as the index date, and only the first eligible readmission was considered for each patient in the cohort. Up to five controls were matched to each case using the methods outlined above for the primary outcome. Each outcome was analyzed separately.

\subsection{Medication Exposure Assessment}

Exposure to medications was assessed using the hospital pharmacy orders available in Health Facts ${ }^{\circledR}$. Patients enrolled in the cohort were required to have received clopidogrel during their first hospitalization for MI. As the 
guidelines by the American College of Cardiology and the American Heart Association recommend a duration of dual antiplatelet therapy for 12 months for most patients [9], all cohort patients were assumed to have maintained clopidogrel treatment for 12 months after initiating treatment at the first hospitalization for MI. Patients were classified as exposed or unexposed to PPIs based on whether they had received any type of PPI during their first hospitalization that qualified for cohort entry. The type of PPI that the patient received during hospitalization was identified for subgroup analyses. PPI exposure status was considered constant for each patient for 12 months post cohort entry or until the patient became a case, whichever occurred earlier. We also assessed the use of medications that may be related to the use of the exposure drugs or the outcomes. These medications were selected based on a review of similar studies in the literature and clinical expert opinion (see electronic supplementary material [ESM] I for the list of comedications). Patient records were searched to determine whether any of these medications were dispensed during the first hospitalization that led to cohort entry. All patients in the cohort were classified as exposed to a specific medication if their medication records included a valid order for that medication; otherwise they were classified as unexposed.

\subsection{Comorbidities and Prior Cardiovascular Procedures}

Electronic hospital admission records of each patient were assessed for the presence of the 30 comorbidities (see ESM I) included in the Elixhauser Comorbidity Measures (ECM) [10]. Patients who had a diagnosis for a condition (as defined in the ECM) during any encounter within the year preceding cohort entry were assumed to have the condition. The following comorbidities, which are related to the exposure or outcome of interest, were included as individual covariates in the regression models described in Sect. 2.6: congestive heart failure, cardiac arrhythmia, valvular disease, pulmonary circulation disorders, peripheral vascular disorders, hypertension, diabetes with complications, peptic ulcer disease, coagulopathy, and blood loss anemia. Values for each covariate were coded as 1 if the patient records included a diagnosis for the condition and 0 if they did not. Similarly, the remaining 20 conditions of the ECM were assessed as either present or absent. The total number of these 20 conditions with which a patient was diagnosed was included in the regression model as a single comorbidity score. (For example, if a patient had been diagnosed with seven of the 20 remaining conditions, the comorbidity score would be equal to 7.)

Patients who had undergone any coronary revascularization (coronary artery bypass grafting), PCI, or carotid revascularization (carotid endarterectomy, stenting, angioplasty, or atherectomy, or carotid bypass) procedures were identified. Having had these procedures was included as a binary covariate in the regression models.

\subsection{Statistical Methods}

Baseline characteristics for the cases and controls for each outcome of interest were compared. The means and standard deviations of continuous variables were reported. The significance of the difference between the cases and controls was determined using Student's t-test when the variables were normally distributed and using the Mann-Whitney $U$ test if the variable was not normally distributed (as determined by the Shapiro-Wilk test). For categorical variables, we reported the corresponding frequencies and percentages and made comparisons using the Chi-squared test.

We conducted case-control analyses for the primary outcome and each of the secondary outcomes. We used conditional logistic regression to estimate the adjusted odds ratio (aOR) and 95\% confidence interval (CI) of hospital readmission for a second MI for PPIs users compared with nonusers. The odds ratios were assessed at 3, 6, and 12 months following cohort entry. Models were further adjusted for additional variables selected based on prior studies and expert clinical opinion, including variables reflecting demographics, smoking, comorbidities, use of comedications, prior cardiovascular procedures, and hospital characteristics. The composite comorbidity score (described in Sect. 2.5) was included as a continuous variable, and the ten individual comorbidities were included as separate covariates in the regression model. Obesity was one of the conditions included in the comorbidity score. The year of cohort entry, the LOS for the first MI encounter, and select characteristics of the admitting hospital (acute status, number of beds, presence of diagnostic catheterization lab, teaching facility) were tested for inclusion in the model; variables that resulted in at least $10 \%$ relative change in the aORs were included in the regression model [11]. Findings for the primary outcome were stratified by age at time of cohort entry (18-64 years; 65-79 years; $80-89$ years) and by the type of PPI that the patient received at cohort entry.

For each of the secondary outcomes (stroke, in-hospital mortality, and the composite endpoint), aORs evaluating PPI use versus non-use were estimated at 3, 6, and 12 months post cohort entry using the methods described. All statistical analyses were completed using SAS software, version 9.4 [12].

\subsection{Negative Control Exposure Drugs}

Hennessy et al. [13] explained how the use of negative control exposure drugs can aid in the interpretation of observed risk estimates. In a typical drug-drug interaction, the 
affected drug is called the object drug (in our case, clopidogrel), whereas the affecting drug is called the precipitant drug (in our case, PPIs). A negative control precipitant drug has similar indications as the precipitant drug under study but is not believed to interact with the object drug. Similarly, a negative control object drug has similar indications as the object drug but has no known pharmacological interactions with the precipitant drug [13]. For this study, we selected one negative control precipitant drug class (H2 receptor antagonists [H2RAs]) and two negative control object drugs (the antiplatelet drugs ticagrelor and prasugrel) to assess the potential for confounding by indication (Fig. 2). We selected from Health Facts ${ }^{\circledR}$ a cohort of patients prescribed each of these antiplatelet drugs using the same methodology described for forming the main study cohort of clopidogrel users (details are presented in ESM I).

\section{Results}

\subsection{Primary Outcome}

We identified a cohort of 52,006 patients from Health Facts ${ }^{\circledR}$ who had been hospitalized for a first MI, received clopidogrel during that hospital encounter, and were discharged alive (Fig. 1). More than half of the cohort (56.3\%) received
PPIs in addition to clopidogrel during their first hospitalization for an MI. The mean age of the cohort was $67.8 \pm$ standard deviation (SD) 12.9 years. The majority of patients were of Caucasian ethnicity (82.1\%) and male (59.3\%). A total of $40 \%$ of the cohort was covered through Medicare, and $15.8 \%$ had private insurance. Medicaid beneficiaries comprised $6.3 \%$ of the cohort and the health insurance provider was missing or unknown in the database for $33.4 \%$ (Table 1). A detailed description of the study cohort is presented in ESM II.

Table 2 presents detailed demographic and clinical characteristics of the matched MI cases and controls included in the analysis. We identified 2932 cases hospitalized for an MI within 12 months of cohort entry. Of these, 2890 cases were matched to 13,933 controls. In total, 42 cases could not be matched to at least one control and were excluded from the analysis: these cases were mainly of non-Caucasian ethnicity (90\%), females $(60 \%)$ and generally aged $<50$ years or $>80$ years. The proportion of females and mean age of patients were not significantly different between cases and controls. However, there was a notable difference in the mean comorbidity index: 5.9 for cases and 4.0 for controls. Cases were more likely than controls to smoke (23.7 vs. $17.8 \%$ ) and to be obese (21.1 vs. $14.8 \%$ ). Cases were also more likely than controls to have experienced a non-ST-elevation MI (NSTEMI) (70.8

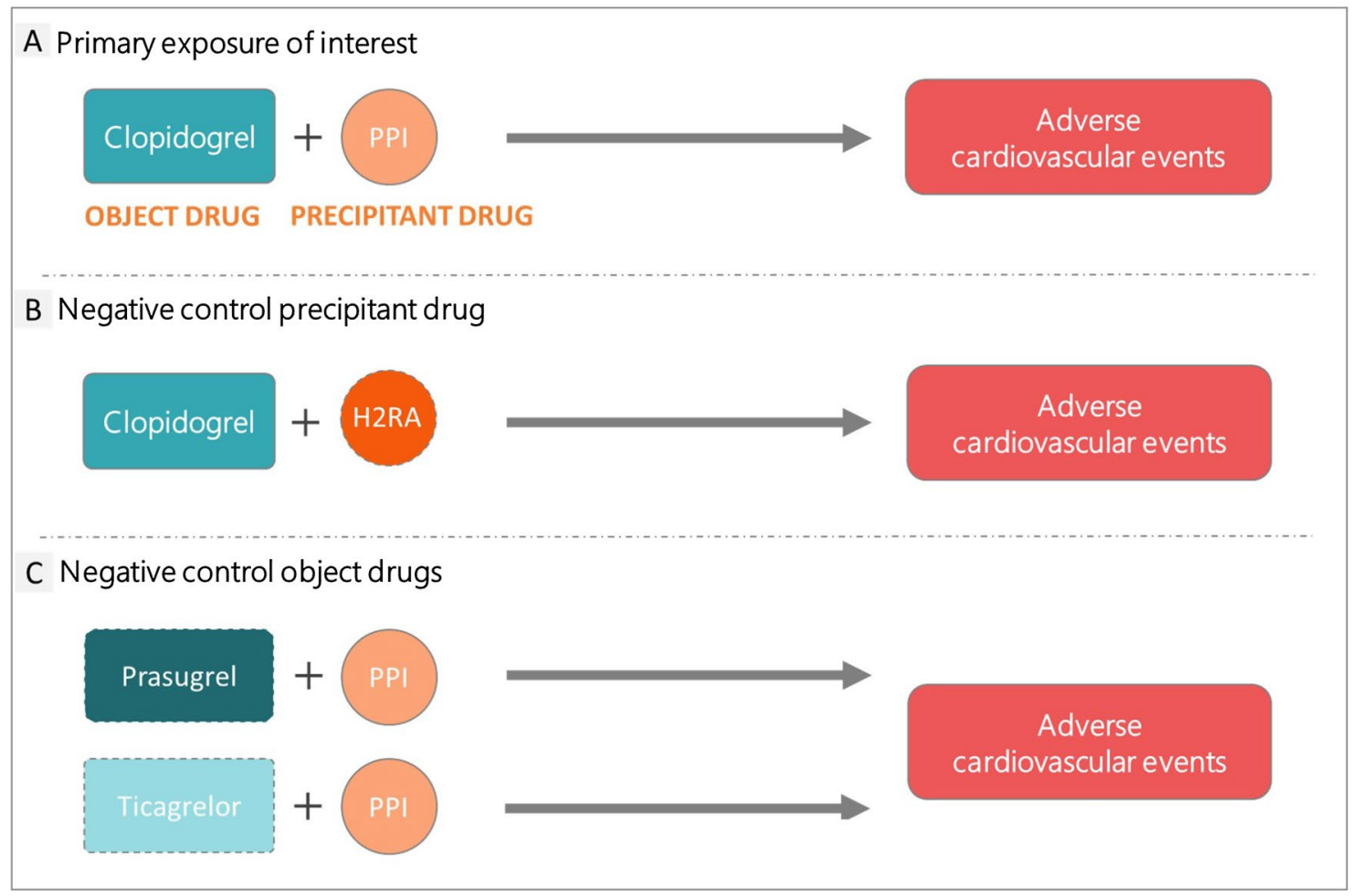

Fig. 2 Illustration of $\mathbf{a}$ the main association of interest; $\mathbf{b}$ H2RAs as a negative control precipitant drug; and $\mathbf{c}$ prasugrel and ticagrelor each as a negative control object drug. $H 2 R A \mathrm{H} 2$ receptor antagonist, $P P I$ proton pump inhibitor 
Table 1 Characteristics of the study cohort comprising clopidogrel users $(N=52,006)$

\begin{tabular}{ll}
\hline Characteristic & Numbers \\
\hline Age (years) & $67.8 \pm 12.9$ \\
Comorbidity score & $4.3 \pm 2.8$ \\
Sex & \\
Male & 59.3 \\
Female & 40.7 \\
Ethnicity & \\
Caucasian & 82.1 \\
African American & 13.3 \\
Other & 4.6 \\
Health insurance status & \\
Medicare & 39.8 \\
Medicaid & 6.3 \\
Uninsured & 4.7 \\
Private & 15.8 \\
Missing/unknown & 33.4 \\
Census region & \\
Midwest & 13.0 \\
Northeast & 46.6 \\
South & 31.5 \\
West & 9.0 \\
\hline
\end{tabular}

Data are presented as mean \pm standard deviation or $\%$

vs. $59.6 \%)$ and to have been diagnosed with congestive heart failure (63.1 vs. $41.0 \%)$, diabetes ( 21.5 vs. $10.8 \%$ ), hypertension ( 22.9 vs. $10.5 \%$ ), or peptic ulcer disease ( 2.3 vs. $1.1 \%$ ) among other diseases. Further, cases were more likely to receive PPIs (58.8 vs. $54.6 \%$ ), aspirin, loop diuretics, calcium channel blockers (CCBs), $\beta$-blockers and antihyperglycemic agents. Controls were more likely to have been hospitalized at hospitals in urban regions than were cases.

Conditional logistic regression performed on the matched MI cases and controls in the primary analyses were adjusted for all the variables in Table 2, excluding PPI use, matching variables (age, sex, and ethnicity) and LOS, health insurance status, acute status of hospital, and hospital bed size. The latter variables were excluded based on empirical testing, as their inclusion had an insignificant impact $(<10 \%$ relative change) on the aOR. Health insurance status was not included in the model, since approximately $30 \%$ of the study sample had missing or unknown health insurance status.

The aOR for experiencing an MI at 12 months post cohort entry for patients who received concurrent clopidogrel-PPI treatment compared with those who received clopidogrel without a PPI was 1.05 (95\% CI 0.96-1.15). Similar aORs were found at 3 and 6 months post cohort entry (Table 3 ). Odds ratios for the negative control, H2RAs, which were expected to be null, were slightly lower than those for PPI use and not statistically significant at the three follow-up periods assessed (Table 3).

aORs were stratified by age group (Table 4). A positive association for recurrent MI with the use of concomitant $\mathrm{PPI} /$ clopidogrel treatment in the group aged 80-89 years (aOR 1.26; 95\% CI 1.05-1.51) was reported, which warrants further investigation. This increased risk was not observed, as expected, with the use of H2RA/clopidogrel in the same age group (aOR 1.01 ; 95\% CI 0.81-1.25). In contrast, a protective effect was estimated for H2RA use among the group aged 18-64 years. Similar findings were observed for the 3- and 6-month follow-up periods.

Subgroup analyses were performed by type of PPI the patient received during the cohort-qualifying hospitalization. The aOR for receiving omeprazole suggested a protective effect for recurrent MI (aOR 0.52; 95\% CI 0.40-0.68), whereas the $\mathrm{aOR}$ for receiving lansoprazole suggested an increased risk of recurrent MI (aOR 4.14; 95\% CI 2.73-6.27). In the current analyses, a relatively low number of MI cases had received lansoprazole $(N=57)$ or omeprazole $(N=85)$, representing 1.9 and $2.9 \%$ of the total number of cases, respectively. Detailed results from this analysis are discussed in ESM II.

\subsection{Secondary Outcomes}

A total of 1551 stroke cases and 2635 in-hospital mortality cases were identified within 12 months of cohort entry, of which 1536 and 2596 cases were matched to 7510 and 12,551 controls, respectively. For the composite outcome, 6493 cases were identified within the same period, of which 6403 cases were matched to 30,957 controls (Fig. 1).

None of the aORs for PPI use versus non-use at 12-month follow-up were statistically significant: stroke (aOR 0.96; 95\% CI 0.85-1.08), all-cause mortality (aOR 1.04; 95\% CI 0.95-1.15), and the composite endpoint (aOR 1.04; 95\% CI 0.98-1.11). ESM II provides a detailed description of the study sample analyzed for each of the secondary outcomes, the aORs at the remaining follow-up periods, and the corresponding ORs for H2RA use vs. non-use.

\subsection{Negative Control Exposure Drugs}

ORs of a recurrent MI using a negative control precipitant drug (H2RAs) and negative control object drugs (ticagrelor and prasugrel) for recurrent MI endpoint are presented in Tables 3 and 5, respectively.

Separate case-control analyses were performed on the cohorts of prasugrel and ticagrelor users. Characteristics of these cohorts in addition to characteristics of the cases and controls for each outcome are provided in ESM II. For prasugrel, aORs for the MI endpoint suggest a lack of association for concomitant treatment with PPIs versus no PPIs (aOR 1.04; 95\% CI 0.67-1.61). ORs were only estimated at 
Table 2 Characteristics of the study sample for myocardial infarction at 12 months follow-up

\begin{tabular}{|c|c|c|c|c|c|}
\hline \multirow{2}{*}{$\frac{\text { Characteristic }}{\text { Age }(\text { years) }}$} & \multicolumn{2}{|c|}{ Cases $(N=2890)$} & \multicolumn{2}{|c|}{ Controls $(N=13,933)$} & \multirow{2}{*}{$\frac{p \text { value }}{0.4}$} \\
\hline & \multicolumn{2}{|l|}{$70.2 \pm 12.1$} & \multicolumn{2}{|l|}{$70.4 \pm 11.7$} & \\
\hline LOS at cohort entry (days) ${ }^{b}$ & \multicolumn{2}{|l|}{$8.3 \pm 7.3$} & \multicolumn{2}{|l|}{$8.0 \pm 7.7$} & 0.1 \\
\hline Comorbidity score & \multicolumn{2}{|l|}{$5.9 \pm 3.0$} & \multicolumn{2}{|l|}{$4.0 \pm 2.8$} & $<0.0001$ \\
\hline \multicolumn{6}{|l|}{ Sex } \\
\hline Females & 1262 & 43.6 & 6029 & 43.3 & 0.7 \\
\hline \multicolumn{6}{|l|}{ Ethnicity } \\
\hline African American & 418 & 14.5 & 1855 & 13.3 & \multirow[t]{3}{*}{$<0.0001$} \\
\hline Caucasian & 2348 & 81.2 & 11,690 & 83.9 & \\
\hline Other & 126 & 4.4 & 394 & 2.8 & \\
\hline \multicolumn{6}{|l|}{ Health insurance status ${ }^{\mathrm{b}}$} \\
\hline Medicare & 1422 & 49.2 & 6799 & 48.8 & \multirow[t]{5}{*}{$<0.0001$} \\
\hline Medicaid & 200 & 7.0 & 853 & 6.1 & \\
\hline Private & 412 & 14.3 & 1560 & 11.2 & \\
\hline Uninsured & 119 & 4.1 & 492 & 3.5 & \\
\hline Missing/unknown & 737 & 25.5 & 4229 & 30.4 & \\
\hline \multicolumn{6}{|l|}{ Census region } \\
\hline Midwest & 320 & 11.1 & 2086 & 15.0 & \multirow[t]{4}{*}{$<0.0001$} \\
\hline Northeast & 1563 & 54.1 & 7314 & 52.5 & \\
\hline South & 789 & 27.3 & 3347 & 24.0 & \\
\hline West & 218 & 7.5 & 1186 & 8.5 & \\
\hline Obesity $^{\mathrm{b}}$ & 611 & 21.1 & 2066 & 14.8 & $<0.0001$ \\
\hline Smoking & 686 & 23.7 & 2480 & 17.8 & $<0.0001$ \\
\hline \multicolumn{6}{|l|}{ MI type } \\
\hline NSTEMI & 2047 & 70.8 & 8306 & 59.6 & \multirow[t]{3}{*}{$<0.0001$} \\
\hline STEMI & 396 & 13.7 & 2969 & 21.3 & \\
\hline Unspecified & 447 & 15.5 & 2658 & 19.1 & \\
\hline \multicolumn{6}{|l|}{$\mathrm{CV}$ procedures } \\
\hline PCI stent & 1394 & 48.2 & 7171 & 51.5 & $<0.01$ \\
\hline CABG & 170 & 5.9 & 982 & 7.1 & 0.02 \\
\hline Carotid revascularization & 49 & 1.7 & 234 & 1.7 & 1.0 \\
\hline Comedications & & & & & \\
\hline Aspirin & 1465 & 50.7 & 5501 & 39.5 & $<0.0001$ \\
\hline ACE inhibitors & 1673 & 57.9 & 8217 & 59.0 & 0.3 \\
\hline$\beta$-Blockers & 2669 & 92.4 & 12,469 & 89.5 & $<0.0001$ \\
\hline $\mathrm{CCBs}$ & 1023 & 35.4 & 4422 & 31.7 & $<0.0001$ \\
\hline Direct vasodilators & 751 & 26.0 & 3330 & 23.9 & 0.02 \\
\hline Loop diuretics & 1784 & 61.7 & 7591 & 54.5 & $<0.0001$ \\
\hline Potassium diuretics & 257 & 8.9 & 1068 & 7.7 & 0.03 \\
\hline Thiazide diuretics & 285 & 9.9 & 1324 & 9.5 & 0.6 \\
\hline Fibrates & 103 & 3.6 & 455 & 3.3 & 0.4 \\
\hline Statins & 2590 & 89.6 & 12,453 & 89.4 & 0.7 \\
\hline GPR antagonists & 537 & 18.6 & 3421 & 24.6 & $<0.0001$ \\
\hline Warfarin & 377 & 13.0 & 2147 & 15.4 & $<0.01$ \\
\hline Antihyperglycemics & 508 & 17.6 & 2099 & 15.1 & $<0.001$ \\
\hline Lytics & 81 & 2.8 & 368 & 2.6 & 0.6 \\
\hline PPIs & 1700 & 58.8 & 7601 & 54.6 & $<0.0001$ \\
\hline H2RAs & 714 & 24.7 & 3850 & 27.6 & $<0.01$ \\
\hline Comorbidities & & & & & \\
\hline Congestive heart failure & 1820 & 63.1 & 5682 & 41.0 & $<0.0001$ \\
\hline Cardiac arrhythmia & 1398 & 48.5 & 5648 & 40.7 & $<0.0001$ \\
\hline Valvular disease & 871 & 30.2 & 2674 & 19.3 & $<0.0001$ \\
\hline
\end{tabular}


Table 2 (continued)

\begin{tabular}{|c|c|c|c|c|c|}
\hline \multirow{2}{*}{$\frac{\text { Characteristic }}{\text { Pulmonary circulation disorders }}$} & \multicolumn{2}{|c|}{ Cases $(N=2890)$} & \multicolumn{2}{|c|}{ Controls $(N=13,933)$} & \multirow{2}{*}{$\begin{array}{l}p \text { value } \\
<0.0001\end{array}$} \\
\hline & 359 & 12.5 & 986 & 7.1 & \\
\hline Peripheral vascular disorders & 793 & 27.5 & 2037 & 14.7 & $<0.0001$ \\
\hline Hypertension & 659 & 22.9 & 1452 & 10.5 & $<0.0001$ \\
\hline Diabetes complicated & 620 & 21.5 & 1493 & 10.8 & $<0.0001$ \\
\hline Peptic ulcer disease & 67 & 2.3 & 156 & 1.1 & $<0.0001$ \\
\hline Coagulopathy & 243 & 8.4 & 815 & 5.9 & $<0.0001$ \\
\hline Blood loss anemia & 77 & 2.7 & 181 & 1.3 & $<0.0001$ \\
\hline \multicolumn{6}{|l|}{ Hospital characteristics } \\
\hline Hospital with full catheterization lab & 2178 & 75.4 & 11,221 & 80.6 & $<0.0001$ \\
\hline Acute care hospital $^{\mathrm{b}}$ & 2883 & 99.8 & 13,930 & 100.0 & $<0.0001$ \\
\hline Hospital in urban location & 2178 & 75.4 & 11,940 & 85.7 & $<0.0001$ \\
\hline Bed size $200-500^{\mathrm{b}}$ & 1142 & 39.5 & 7497 & 53.8 & $<0.0001$ \\
\hline Bed size $<200^{b}$ & 456 & 15.8 & 1460 & 10.5 & \\
\hline Bed size $>500^{\mathrm{b}}$ & 1290 & 44.7 & 4973 & 35.7 & \\
\hline
\end{tabular}

Data are presented as mean \pm standard deviation (comparison made using Mann-Whitney $U$ test) or $n(\%)$ unless otherwise indicated

$A C E$ angiotensin-converting enzyme, $C A B G$ coronary artery bypass grafting, $C C B s$ calcium channel blockers, $C V$ cardiovascular, GPR glycoprotein IIb/IIIa receptor, H2RA $\mathrm{H} 2$ receptor antagonist, LOS length of stay, $M I$ myocardial infarction, NSTEMI non-ST-elevation myocardial infarction, $P C I$ percutaneous coronary intervention, $P P I$ proton pump inhibitor, STEMI ST-elevation myocardial infarction

${ }^{a}$ Matching variable

${ }^{\mathrm{b}}$ Variable not included in regression models; obesity was included in the comorbidity score and not as an individual covariate

Table 3 Adjusted odds ratios for the risk of the outcomes with proton pump inhibitor use (vs. non-use) and H2RA use (vs. non-use) among clopidogrel users at 3-, 6-, and 12-month follow-up

\begin{tabular}{|c|c|c|c|c|}
\hline Endpoint and follow-up & Matched cases/controls & Unmatched cases & $\begin{array}{l}\text { PPI use vs. non-use, OR } \\
(95 \% \mathrm{CI})\end{array}$ & $\begin{array}{l}\text { H2RA use vs. non-use } \\
\text { (negative control), OR (95\% } \\
\text { CI) }\end{array}$ \\
\hline \multicolumn{5}{|l|}{ MI } \\
\hline 3 months & $1569 / 7573$ & 24 & $1.08(0.95-1.23)$ & $0.88(0.76-1.02)$ \\
\hline 6 months & $2220 / 10,684$ & 32 & $1.05(0.95-1.17)$ & $0.9(0.81-1.03)$ \\
\hline 12 months & $2890 / 13,933$ & 42 & $1.05(0.96-1.15)$ & $0.93(0.84-1.04)$ \\
\hline \multicolumn{5}{|l|}{ In-hospital mortality } \\
\hline 3 months & $1418 / 6845$ & 22 & $1.13(0.98-1.29)$ & $1.05(0.91-1.22)$ \\
\hline 6 months & $1933 / 9348$ & 28 & $1.11(0.99-1.24)$ & $1.01(0.89-1.15)$ \\
\hline 12 months & $2596 / 12,551$ & 39 & $1.04(0.95-1.15)$ & $0.93(0.83-1.04)$ \\
\hline \multicolumn{5}{|l|}{ Stroke } \\
\hline 3 months & $764 / 3717$ & 6 & $0.92(0.77-1.10)$ & $0.87(0.71-1.06)$ \\
\hline 6 months & $1101 / 5377$ & 8 & $0.96(0.83-1.12)$ & $0.86(0.73-1.02)$ \\
\hline 12 months & $1536 / 7510$ & 15 & $0.96(0.85-1.08)$ & $0.84(0.73-0.97)$ \\
\hline \multicolumn{5}{|l|}{ Composite endpoint } \\
\hline 3 months & $3493 / 16,902$ & 52 & $1.07(0.99-1.17)$ & $0.95(0.87-1.05)$ \\
\hline 6 months & $4838 / 23,384$ & 67 & $1.06(0.99-1.14)$ & $0.96(0.88-1.03)$ \\
\hline 12 months & $6403 / 30,957$ & 90 & $1.04(0.98-1.11)$ & $0.93(0.87-1.00)$ \\
\hline
\end{tabular}

$C I$ confidence interval, $H 2 R A \mathrm{H} 2$ receptor antagonist, $M I$ myocardial infarction, $O R$ odds ratio

12 months post cohort entry because of the relatively small number of cases for the earlier follow-up periods. aORs for the secondary outcomes also suggest a lack of association for combined PPI treatment with prasugrel. Similarly, we observed no association between receiving PPIs in combination with ticagrelor and experiencing an MI (aOR 0.96; 
Table 4 Adjusted odds ratios stratified by age for proton pump inhibitor use vs. non-use and myocardial infarction recurrence among clopidogrel users at 12 months post cohort entry

\begin{tabular}{|c|c|c|c|}
\hline \multirow{2}{*}{$\begin{array}{l}\text { Age group, } \\
\text { years }\end{array}$} & \multirow{2}{*}{$\begin{array}{l}\text { Number of } \\
\text { cases/con- } \\
\text { trols }\end{array}$} & \multicolumn{2}{|l|}{ OR $(95 \% \mathrm{CI})$} \\
\hline & & $\begin{array}{l}\text { PPI use vs. } \\
\text { non-use }\end{array}$ & $\begin{array}{l}\text { H2RA use vs. } \\
\text { non-use (negative } \\
\text { control) }\end{array}$ \\
\hline $18-64$ & $342 / 3980$ & $0.96(0.80-1.14)$ & $0.71(0.58-0.88)$ \\
\hline $65-79$ & $1048 / 5065$ & $0.97(0.83-1.14)$ & $1.07(0.89-1.28)$ \\
\hline $80-89$ & $767 / 3702$ & $1.26(1.05-1.51)$ & $1.01(0.81-1.25)$ \\
\hline
\end{tabular}

$C I$ confidence interval, $H 2 R A \mathrm{H} 2$ receptor antagonist, $O R$ odds ratio, $P P I$ proton pump inhibitor

95\% CI 0.69-1.33) or experiencing either of the secondary endpoints (Table 5).

However, compared with the aORs for clopidogrel use in combination with PPIs, wider CIs were observed around the OR's point estimates because of the relatively small number of cases in each analysis. The point estimates were similar in magnitude between the two cohorts (prasugrel and ticagrelor users), except for stroke among prasugrel users, where the point estimate was 0.52 among prasugrel users and 1.24 among ticagrelor users. Neither OR achieved statistical significance.

\section{Discussion}

Since the first reports of a potential interaction between clopidogrel and PPIs [14], many observational studies have investigated the potential clinical impact among patients receiving concomitant treatment with both drugs and have arrived at inconsistent findings. In the present nested case-control study, we analyzed EMRs that represented realworld data over a 17-year period for a cohort of patients who initiated clopidogrel treatment after experiencing an MI. Overall, findings do not support an association between the concomitant use of clopidogrel and PPIs and an increased risk for MI readmission, stroke, all-cause mortality, or a composite endpoint.

Based on the biological interaction reported between PPIs and clopidogrel, PPIs have the potential to compete for the active site of the CYP2C19, the enzyme mainly responsible for converting clopidogrel into its active form. It is hypothesized that clopidogrel's antiplatelet activity is attenuated by way of competitive inhibition, potentially putting patients taking both medications at increased risk of a cardiovascular event relative to clopidogrel-treated patients not receiving PPIs.

Although findings do not support an association between combined treatment recurrences of an MI, we did find a possible increased risk of MI recurrence among patients aged $\geq 80$ years: patients in this age group who received a PPI appeared to be more susceptible to experiencing a second MI than were patients not receiving PPI (aOR 1.26; 95\% CI 1.05-1.51). Although this may be a spurious association, there are several possible explanations why this association was detected in this age group. First, given the increased number of comorbidities that occur with age [15], elderly subjects are likely to be on multiple medications in addition to PPIs and may therefore be more susceptible to experiencing drug-drug interactions [16], including CYP-mediated drug-drug interactions. Further, patients aged $>80$ years may be more vulnerable to adverse drug events because of metabolic and physiologic changes that come with increased age, and thus the clopidogrel-PPI interaction demonstrated in mechanistic studies may be clinically meaningful among elderly patients. Further investigation of a potentially clinically significant interaction between PPIs and clopidogrel among elderly patients is warranted.

Our findings are consistent with prior studies that have reported a lack of an association between concomitant treatment and an increased risk for an MI relative to treatment with clopidogrel alone [17-21]. This finding supports the idea that, although mechanistic studies show that PPIs have the potential to attenuate the pharmacodynamic effects of clopidogrel, it is possible that this does not translate into adverse clinical outcomes among the majority of the patients with ACS.

O'Donoghue et al. [17] reported a significantly lower inhibition of platelet aggregation among patients on combined treatment relative to patients on clopidogrel alone. These same authors performed a post-hoc analysis of the TRITON-TIMI 38 trial and did not detect an association between PPI use and the composite endpoint of
Table 5 Adjusted odd ratios of outcomes among ticagrelor and prasugrel users for proton pump inhibitor use vs. non-use at 12 months post cohort entry

\begin{tabular}{llllll}
\hline Outcome & \multicolumn{2}{l}{ Ticagrelor users } & & \multicolumn{2}{l}{ Prasugrel users } \\
\cline { 2 - 3 } & Cases/controls & OR $(95 \% \mathrm{CI})$ & & Cases/controls & OR (95\% CI) \\
\hline MI & $251 / 1111$ & $0.96(0.69-1.33)$ & & $173 / 811$ & $1.04(0.67-1.61)$ \\
In-hospital mortality & $131 / 548$ & $0.82(0.50-1.35)$ & $75 / 340$ & $0.85(0.36-2.01)$ \\
Stroke & $105 / 446$ & $1.24(0.68-2.27)$ & & $70 / 330$ & $0.52(0.19-1.42)$ \\
Composite endpoint & $454 / 1960$ & $0.93(0.73-1.18)$ & & $304 / 1417$ & $1.03(0.74-1.42)$ \\
\hline
\end{tabular}

CI confidence interval, $M I$ myocardial infarction, $O R$ odds ratio 
cardiovascular mortality, MI, or stroke among clopidogrel users. The authors speculated that, despite the attenuation of the antiplatelet effect of clopidogrel in vitro, the effect may be insufficient to lead to an increased risk of adverse clinical events.

Various studies have reported positive associations between PPI use and adverse cardiovascular events among clopidogrel users but have attributed the observed findings to unmeasured confounders [18, 19, 22, 23]. In this study, we matched cases and controls on important characteristics and included an extensive list of variables, including comedications and comorbidities, to adjust for possible confounders that may affect the exposure or the outcome. Of note is the inclusion of the comorbidity index, which was based on the ECM. Conditions related to the outcome or exposure were removed and put directly into the regression model. We also included the smoking status, a well-documented risk factor for MI, of patients referred from the presence of ICD codes for tobacco use (ICD9 305.1; ICD-10: F17.x and Z72.0). Smoking may also be associated with PPI use, as smokers are at increased risk of developing peptic ulcers and may therefore be more likely to receive PPIs [24].

ORs for the risk of MI stratified by the type of PPI patients received during their cohort-qualifying hospitalization suggested a protective effect for omeprazole and a harmful effect for lansoprazole. PPI types differ in their ability to inhibit CYP2C19 (based on their metabolism), where omeprazole is believed to be the strongest inhibitor among PPIs [25]. If the competitive inhibition caused by omeprazole translated into a clinically significant effect, we would expect to see an increased risk of MI among patients being treated with both clopidogrel and omeprazole. Hence, in the absence of plausible biological mechanisms underlying these findings, it is difficult to interpret the observed ORs. It is possible that the reported associations represent false-positive results in the presence of small sample sizes. (In this study, only 56 and 84 MI cases received lansoprazole and omeprazole, respectively.) Further investigations involving larger sample sizes would be helpful in clarifying these findings.

\subsection{Negative Control Exposure Drugs}

Lipsitch et al. [26] stressed that negative controls should be applied more extensively in epidemiological studies to detect confounding from unmeasured variables. The authors further explained that "the essential purpose of a negative control is to reproduce a condition that cannot involve the hypothesized causal mechanism but is very likely to involve the same sources of bias that may have been present in the original association."

Figure 2 illustrates how, in this study, we assessed and compared the aOR corresponding to the main exposures of interest (clopidogrel and PPIs) to those for negative control exposure drugs. Ticagrelor and prasugrel are both common antiplatelet agents; however, prasugrel has a lower dependency (than clopidogrel) on the CYP2C19 pathway for activation, whereas ticagrelor is taken in its active form and does not require activation in the body. As such, null associations are expected between exposure to these drugs in combination with PPIs and adverse cardiovascular events. (If elevated risks were observed, they may then be attributed to residual confounding.) aORs for H2RA use versus non-use among clopidogrel users were evaluated for all endpoints and were comparable to the aOR for PPIs, suggesting that potential confounders that could significantly impact the findings have been adequately controlled for.

The use of H2RAs as a negative control is not new in the context of PPIs. A Canadian study [27] reported a twofold higher risk of MI recurrence in both elderly patients who had initiated PPIs (vs. no PPIs) as well as in patients who had initiated H2RAs (vs. no H2RA). Since H2RAs have no known causal link to MIs, the authors dismissed the presence of an association and interpreted their findings as due to bias or confounding. Similar interpretations of findings were reported by Goodman et al. [28] and Charlot et al. [19]. Moreover, given the absence of a randomized controlled trial (RCT) where PPI exposure is randomized among patients, Kwok and Loke [29] suggested an RCT with H2RAs serving as an appropriate control exposure among clopidogreltreated patients should be conducted.

ORs reported in this study for the concomitant use of either of the negative control object drugs, ticagrelor and prasugrel, with PPIs also do not show any increased risk of MI compared with non-use of PPIs. Our findings are in agreement with prior observational studies that have assessed clinical endpoints and have reported similar findings among ticagrelor users [30, 31] and prasugrel users in a post-hoc analysis of the TRITON-TIMI 38 trial [17]. Goodman et al. [28] reported a positive association (adjusted hazard ratio 1.24; 95\% CI 1.07-1.45) between PPIs and the composite outcome (cardiovascular death, MI, or stroke) among patients with ACS receiving ticagrelor based on a post-hoc analysis of the PLATO trial. However, they also reported a lack of association for the individual outcomes. The authors dismissed the possibility of a causal association and attributed the elevated risk they identified to confounding by indication. Their conclusion was based on the observation of higher event rates among patients treated with a PPI, which were independent of receiving an antiplatelet. Our observed null associations for H2RA use versus nonuse suggest that the statistical analyses-which adjusted for numerous covariates - had adequately controlled for residual confounding.

Studies on platelet reactivity have also been performed on these two antiplatelet agents. Storey et al. [32] reported 
no difference in platelet reactivity among patients receiving ticagrelor with or without PPIs. Similarly, no difference in platelet reactivity or platelet inhibition was reported among patients who received prasugrel with or without PPIs [33] and specifically lansoprazole [34].

Prasugrel requires hepatic metabolism to transform it to its active form; however, CYP2C19 has a relatively small contribution and is not predominant in the bioactivation process [25]. Further, a large proportion of prasugrel is believed to be activated by intestinal CYP3A enzymes [33]. Ticagrelor, on the other hand, is a direct-acting drug that does not require bioactivation in the body. Hence, the potential for PPIs to interact with both prasugrel and ticagrelor is low compared with clopidogrel, and interactions of these antiplatelets with PPIs have not been reported [35].

\subsection{Limitations}

The use of EMRs collected for purposes other than epidemiological research, while providing large sample sizes, introduces some limitations. Drug exposure was assessed at the time of admission for a first MI and was assumed to be constant during the 12-month follow-up period or until the patient experienced an outcome of interest (whichever occurred first). During follow-up, we did not have information on patients' adherence to the medication regimen they received at cohort entry, including whether they may have switched between different antiplatelet agents, discontinued any of the drugs, or initiated new medications. These factors could not be accounted for in the analysis and could potentially bias the results. However, although switching between antiplatelet agents remains a possibility for some patients, a large multicenter study reported that over $90 \%$ of patients who were discharged on dual antiplatelet therapy (DAPT) did not switch to another antiplatelet during the first 3 months following initiation, and $76 \%$ of patients did not switch during the first 12 months following DAPT initiation [36]. If we assume similar rates of switching occurred in the current study sample, then misclassification of exposure, particularly in the effect estimates at 3 months following clopidogrel initiation, would not be expected to significantly affect the reported results. To minimize the potential for including patients who are likely to switch antiplatelet agents, we excluded patients who had received more than one of three common antiplatelets (clopidogrel, prasugrel, and ticagrelor) during their first hospitalization.

Further, information on the use of over-the-counter drugs was not available for use in our analyses. PPIs and aspirin are available without prescription in the USA, which may result in exposure misclassification for these medications. It is possible for patients who received PPIs during hospitalization to discontinue them later, or for patients who had not received PPIs to initiate them post cohort entry. This type of misclassification - if nondifferential and based on a dichotomous exposure-has the potential to bias results toward the null and would be expected to be more pronounced with time since exposure assessment (at cohort entry in this study) [37, 38]. Comparison of the 12-month ORs for the primary and secondary endpoints with those at 3 and 6 months did not reveal substantial differences to indicate decreasing risk with shorter duration of use. This suggests that if misclassification of exposure was present, it did not introduce significant bias in our findings.

The potential for misclassification of outcome also exists as the cohort entry eligibility and the identification of MI cases was based on ICD diagnosis codes. Another limitation is that Health Facts ${ }^{\circledR}$ does not capture potential cases admitted to health facilities that are not part of the Cerner network or deaths that occur outside hospitals.

Finally, some variables used in the analysis had missing data. Patients with missing information on ethnicity (2.67\%), sex $(0.15 \%)$, and age $(0.80 \%)$ were excluded from cohort entry. The proportions of these missing observations were small, and their exclusion was unlikely to have biased our findings. Further, approximately $30 \%$ of the cohort had missing or unknown values for health insurance status, a variable that can serve as an indicator of socioeconomic status. We decided not to include this variable in the regression model and performed a sensitivity analysis by assessing the effect of this variable when included or excluded from the models. Results showed that incorporation of health insurance status did not have a notable effect on the ORs, where the percent change in ORs was between 1.25 and $1.05 \%$ compared with results excluding the variable. This was consistent across all four outcomes at the three follow-up periods assessed (ESM II).

\subsection{Strengths}

Although the examined associations have been extensively studied over the last decade, the clinical significance of the potential association remains relevant today as clopidogrel and PPIs are commonly coprescribed to patients with ACS. The current study has many strengths, including a large geographically diverse cohort of US patients from over 500 health centers and covering the 2000-2016 period. The cohort of clopidogrel users was selected from health records of over 69 million US patients residing across all census regions of the USA. The Health Facts ${ }^{\circledR}$ database is rich in real-world clinical information that allowed us to adjust our findings for many potential confounders. In particular, the reported ORs were adjusted for smoking and obesity; smoking was included as a separate dichotomous covariate, and obesity was included in the comorbidity score. Neither variable is commonly captured in administrative databases, and this has been reported as a limitation in similar studies [19, 
$22,23,39]$. The large study sample also allowed us to further stratify the findings by age groups and perform sensitivity analyses using laboratory data.

The associations between adverse cardiovascular events and PPI use among ticagrelor or prasugrel users contribute useful information to the current body of literature on these agents, as our earlier systematic review showed that reported findings on this association are scarce.

\section{Conclusion}

Findings from this case-control study nested in a large retrospective cohort of patients who had experienced an MI and received clopidogrel do not provide evidence of an association between PPI use and MI, stroke, or all-cause mortality compared with nonuse of PPIs. An increased risk for MI was detected among patients aged 80-89 years but not in younger age groups. Although the interpretation of this finding is unclear at this time, it may warrant further investigation in subsequent studies. aORs for PPI use versus nonuse were similar in magnitude to those for H2RA use versus non-use among clopidogrel users. In summary, this study adds to the literature findings in support of a lack of clinical impact of combined treatment with clopidogrel and PPIs from a relatively large and diverse cohort using statistical analyses controlling for a wide range of covariates. Although there is potential for a biological interaction between clopidogrel and PPIs, this interaction likely does not lead to detectable adverse clinical effects.

\section{Compliance with ethical standards}

Conflict of interest DK is the Natural Sciences and Engineering Research Council of Canada Chair in Risk Science at the University of Ottawa, a peer reviewed university-industry research partnership program. DK and DRM, respectively, serve as Chief Risk Scientist and Chief Medical Officer for Risk Sciences International (RSI) (www. risksciences.com), a Canadian company established in 2006 in partnership with the University of Ottawa to provide consulting services in risk science to both public and private sector clients. To date, RSI has not conducted work on PPIs or antiplatelet agents that are the subject of the present paper. AW is a clinical cardiologist and has treated patients with cardiovascular diseases, including myocardial infarction, using PPIs and antiplatelet agents, including clopidogrel. NB has served as a consultant to RSI. NF has served as a consultant to RSI and international clients on issues unrelated to those discussed in the present paper. $\mathrm{NH}$ is a senior epidemiologist with the Public Health Agency of Canada working on matters unrelated to the subject of the present paper. YF, FM, SWW, DSM, and DRM have no conflicts of interest that are directly relevant to the content of this article.

Availability of data and material The data that support the findings of this study are available from Cerner Health Facts@. We sought permission from Cerner to perform the current study. Restrictions apply to the availability of these data, and they are not publicly available.
Funding This study was funded by a Canadian Institutes of Health Research (CIHR) Doctoral Research Award (to NF) and by the McLaughlin Center for Population Health Risk Assessment, University of Ottawa, Canada.

Open Access This article is licensed under a Creative Commons Attribution-NonCommercial 4.0 International License, which permits any non-commercial use, sharing, adaptation, distribution and reproduction in any medium or format, as long as you give appropriate credit to the original author(s) and the source, provide a link to the Creative Commons licence, and indicate if changes were made. The images or other third party material in this article are included in the article's Creative Commons licence, unless indicated otherwise in a credit line to the material. If material is not included in the article's Creative Commons licence and your intended use is not permitted by statutory regulation or exceeds the permitted use, you will need to obtain permission directly from the copyright holder. To view a copy of this licence, visit http://creativecommons.org/licenses/by-nc/4.0/.

\section{References}

1. Pelliccia F, Rollini F, Marazzi G, Greco C, Gaudio C, Angiolillo DJ. Drug-drug interactions between clopidogrel and novel cardiovascular drugs. Eur J Pharmacol. 2015;765:332-6. https://doi. org/10.1016/j.ejphar.2015.08.059.

2. Bhatt B, Scheiman J, Abraham N, Antman E, Chan F, Furberg F, et al. ACCF/ACG/AHA 2008 Expert Consensus Document on Reducing the Gastrointestinal Risks of Antiplatelet Therapy and NSAID Use. A Report of the American College of Cardiology Foundation Task Force on Clinical Expert Consensus Documents. J Am Coll Cardiol. 2008;52:1502-17. https://doi.org/10.1016/j. jacc.2008.08.002.

3. Kazui M, Nishiya Y, Ishizuka T, Hagihara K, Farid NA, Okazaki $\mathrm{O}$, et al. Identification of the human cytochrome P450 enzymes involved in the two oxidative steps in the bioactivation of clopidogrel to its pharmacologically active metabolite. Drug Metab Dispos. 2010;38:92-9. https://doi.org/10.1124/dmd.109.029132.

4. Farhat N, Fortin Y, Haddad N, Birkett N, Mattison D, Momoli $\mathrm{F}$, et al. Systematic review and meta-analysis of adverse cardiovascular events associated with concomitant use of proton pump inhibitors and antiplatelet agents. Crit Rev Toxicol. 2019. https:// doi.org/10.1080/10408444.2019.1583167.

5. Kim SC, Solomon DH, Rogers JR, Gale S, Klearman M, Sarsour $\mathrm{K}$, et al. Cardiovascular safety of tocilizumab versus tumor necrosis factor inhibitors in patients with rheumatoid arthritis: a multidatabase cohort study. Arthritis Rheumatol. 2017;69:1154-64. https://doi.org/10.1002/art.40084.

6. Kiyota Y, Schneeweiss S, Glynn RJ, Cannuscio CC, Avorn J, Solomon DH. Accuracy of medicare claims-based diagnosis of acute myocardial infarction: estimating positive predictive value on the basis of review of hospital records. Am Heart J. 2004;148:99-104. https://doi.org/10.1016/J.AHJ.2004.02.013.

7. Lubin JH, Gail MH. Biased selection of controls for case-control analyses of cohort studies. Biometrics. 1984;40:63. https://doi. org/10.2307/2530744.

8. Robins JM, Gail MH, Lubin JH. More on biased selection of controls for case-control analyses of cohort studies. Biometrics. 1986;42:293. https://doi.org/10.2307/2531050.

9. Levine GN, Bates ER, Bittl JA, Brindis RG, Fihn SD, Fleisher LA, et al. 2016 ACC/AHA guideline focused update on duration of dual antiplatelet therapy in patients with coronary artery disease: a report of the American College of Cardiology/American Heart 
Association Task Force on Clinical Practice Guidelines. Circulation. 2016;134:e123-e155155. https://doi.org/10.1161/CIR.00000 00000000404 .

10. Quan H, Sundararajan V, Halfon P, Fong A, Burnand B, Luthi JC, et al. Coding algorithms for defining comorbidities in ICD-9-CM and ICD-10 administrative data. Med Care. 2005;43:1130-9. https ://doi.org/10.1097/01.mlr.0000182534.19832.83.

11. Greenland S. Modeling and variable selection in epidemiologic analysis. Am J Public Health. 1989;79:340-9. https://doi. org/10.2105/ajph.79.3.340.

12. SAS Institute Inc. SAS Version 9.4 software. Cary, NC.

13. Hennessy S, Leonard CE, Gagne JJ, Flory JH, Han X, Brensinger $\mathrm{CM}$, et al. Pharmacoepidemiologic methods for studying the health effects of drug-drug interactions. Clin Pharmacol Ther. 2016;99:92-100. https://doi.org/10.1002/cpt.277.

14. Gilard M, Arnaud B, Le Gal G, Abgrall J, Boschat J. Influence of omeprazole on the antiplatelet action of clopidogrel associated to aspirin. J Thromb Haemost. 2006;4:2508-9.

15. Kantor ED, Rehm CD, Haas JS, Chan AT, Giovannucci EL. Trends in prescription drug use among adults in the United States from 1999-2012. JAMA. 2015;314:1818-31. https://doi. org/10.1001/jama.2015.13766.

16. Maher RL, Hanlon J, Hajjar ER. Clinical consequences of polypharmacy in elderly. Expert Opin Drug Saf. 2014;13:57-655. https ://doi.org/10.1517/14740338.2013.827660.

17. O’Donoghue ML, Braunwald E, Antman EM. Pharmacodynamic effect and clinical efficacy of clopidogrel and prasugrel with or without a proton-pump inhibitor: an analysis of two randomised trials. Lancet. 2009;374(9694):989-97.

18. Rassen JA, Choudhry NK, Avorn J, Schneeweiss S. Cardiovascular outcomes and mortality in patients using clopidogrel with proton pump inhibitors after percutaneous coronary intervention or acute coronary syndrome. Circulation. 2009;120:2322-9. https ://doi.org/10.1161/CIRCULATIONAHA.109.873497.

19. Charlot M, Ahlehoff O, Norgaard ML, Jørgensen CH, Sørensen R. Proton-pump inhibitors are associated with increased cardiovascular risk independent of clopidogrel use. Ann Intern Med. 2010;153:379. https://doi.org/10.7326/0003-4819-153-6-201009210-00005.

20. Sarafoff N, Sibbing D, Sonntag U, Ellert J, Schulz S, Byrne R, et al. Risk of drug-eluting stent thrombosis in patients receiving proton pump inhibitors. Thromb Haemost. 2010;104:626-32. https://doi.org/10.1160/TH09-11-0800.

21. Douglas IJI, Evans SSJW, Hingorani A, Grosso A, Timmis A, Hemingway $\mathrm{H}$, et al. Clopidogrel and interaction with proton pump inhibitors: comparison between cohort and within person study designs. BMJ (Online). 2012;345:1-14. https://doi. org/10.1136/bmj.e4388.

22. Stockl KM, Le L, Zakharyan A, Harada ASM, Solow BK, Addiego JE, et al. Risk of rehospitalization for patients using clopidogrel with a proton pump inhibitor. Arch Intern Med. 2010;170:704-10. https://doi.org/10.1001/archinternmed.2010.34.

23. Mahabaleshwarkar RK, Yang Y, Datar MV, Bentley JP, Strum MW, Banahan BF, et al. Risk of adverse cardiovascular outcomes and all-cause mortality associated with concomitant use of clopidogrel and proton pump inhibitors in elderly patients. Curr Med Res Opin. 2013;29:315-23. https://doi.org/10.1185/03007995.2013.772051.

24. Eastwood GL. The role of smoking in peptic ulcer disease. J Clin Gastroenterol. 1988;10(Suppl 1):S19-23.

25. Scott SA, Owusu Obeng A, Hulot J-S. Antiplatelet drug interactions with proton pump inhibitors. Expert Opin Drug Metab Toxicol. 2014;10:175-89. https://doi.org/10.1517/17425 255.2014.856883.

26. Lipsitch M, Tchetgen E, Cohen T. Negative controls: a tool for detecting confounding and bias in observational studies. Epidemiology. 2010;21:383-8. https://doi.org/10.1097/EDE.0b013e3181 d61eeb.Negative.
27. Juurlink DN, Dormuth CR, Huang A, Hellings C, Paterson $\mathrm{JM}$, Raymond C, et al. Proton pump inhibitors and the risk of adverse cardiac events. PLoS ONE. 2013;8:e84890. https://doi. org/10.1371/journal.pone.0084890.

28. Goodman SG, Clare R, Pieper KS, Nicolau JC, Storey RF, Cantor WJ, et al. Association of proton pump inhibitor use on cardiovascular outcomes with clopidogrel and ticagrelor: insights from the platelet inhibition and patient outcomes trial. Circulation. 2012;125:978-86. https://doi.org/10.1161/CIRCULATIO NAHA.111.032912.

29. Kwok CS, Loke YK. Meta-analysis: the effects of proton pump inhibitors on cardiovascular events and mortality in patients receiving clopidogrel. Aliment Pharmacol Ther. 2010;31:810-23. https://doi.org/10.1111/j.1365-2036.2010.04247.x.

30. Yan Y, Wang X, Fan J-Y, Nie S-P, Raposeiras-Roubín S, Abu-Assi $\mathrm{E}$, et al. Impact of concomitant use of proton pump inhibitors and clopidogrel or ticagrelor on clinical outcomes in patients with acute coronary syndrome. J Geriatr Cardiol JGC. 2016;13:209-17. https://doi.org/10.11909/j.issn.1671-5411.2016.03.007.

31. Hoedemaker NPG, Damman P, Ottervanger JP, Dambrink JHE, Gosselink ATM, Kedhi E, et al. Trends in cardiovascular and bleeding outcomes in acute coronary syndrome patients treated with or without proton-pump inhibitors during the introduction of novel P2Y12 inhibitors : a five-year experience from a singlecentre observational registry. Eur Heart J Cardiovasc Pharmacother. 2018;5(3):127-38. https://doi.org/10.1093/ehjcvp/pvy030.

32. Storey RF, Angiolillo DJ, Patil SB, Desai B, Ecob R, Husted S, et al. Inhibitory effects of ticagrelor compared with clopidogrel on platelet function in patients with acute coronary syndromes: The PLATO (PLATelet inhibition and patient Outcomes) PLATELET substudy. J Am Coll Cardiol. 2010;56:1456-62. https://doi. org/10.1016/j.jacc.2010.03.100.

33. Nicolau JC, Bhatt DL, Roe MT, Lokhnygina Y, Neely B, Corbalán $\mathrm{R}$, et al. Concomitant proton-pump inhibitor use, platelet activity, and clinical outcomes in patients with acute coronary syndromes treated with prasugrel versus clopidogrel and managed without revascularization: Insights from the Targeted Platelet Inhibition to Cl. Am Heart J. 2015;170(683-94):e3. https://doi.org/10.1016/j. ahj.2015.05.017.

34. Collet JP, Hulot JS, Abtan J, Anzaha G, Kerneis M, Silvain J, et al. Prasugrel but not high dose clopidogrel overcomes the lansoprazole neutralizing effect of P2Y12inhibition: results of the randomized DOSAPI study. Eur J Clin Pharmacol. 2014;70:1049-57. https://doi.org/10.1007/s00228-014-1710-1.

35. Bolek T, Samoš M, Škorňová I, Kovář F, Galajda P, Staško J, et al. Proton pump inhibition in patients treated with novel antithrombotic drugs: should we worry about thrombosis? J Cardiovasc Pharmacol. 2018;72(1):71-6. https://doi.org/10.1097/fjc.00000 00000000593.

36. Bueno H, Pocock S, Danchin N, Annemans L, Gregson J, Medina $\mathrm{J}$, et al. International patterns of dual antiplatelet therapy duration after acute coronary syndromes. Heart (British Cardiac Society). 2017;103:132-8. https://doi.org/10.1136/heartjnl-2016-309509.

37. Rothman KJ. Epidemiology: an introduction. Oxford: Oxford University Press; 2002.

38. Schneeweiss S. A basic study design for expedited safety signal evaluation based on electronic healthcare data. Pharmacoepidemiol Drug Saf. 2010;19:858-68. https://doi.org/10.1002/ nbm.3066.

39. Schmidt M, Johansen MB, Robertson DJ, Maeng M, Kaltoft A, Jensen LO, et al. Concomitant use of clopidogrel and proton pump inhibitors is not associated with major adverse cardiovascular events following coronary stent implantation. Alimentary Pharmacol Ther. 2012;35:165-74. https://doi.org/10.111 $1 / \mathrm{j} .1365-2036.2011 .04890 . x$. 